\title{
Mesures sur carottes de sondage de quelques propriétés physiques de bois de hêtre à fortes contraintes de croissance
}

\author{
Voïchita BUCUR \\ I.N.R.A., Station de Recherches sur la Qualité des Bois \\ Centre de Recherches forestières de Nancy. Champenoux, F 54280 Scichamps
}

\section{Résumé}

Le retrait dı bois de hêtre normal et à fortes contraintes de croissance a été étudié sur des carottes de sondage, en liaison avec la densité radiographique des gros rayons ligneux, les caractéristiques des fibres, l'angle des microfibrilles et l'infradensité.

L'étude fine, cerne par cerne, du cœur vers l'écorce, a montré que le bois à fortes contraintes de croissance analysé dans cette expérience se caractérise par une infradensité, un retrait longtitudinal et un nombre de gros rayons ligneux par $\mathrm{cm}^{2}$ légèrement supérieurs à ceux du bois normal.

En revanche, la densité des rayons ligneux et l'angle des microfibrilles du bois à fortes contraintes sont plus faibles que ceux du bois normal.

Mots clés : Carottes de sondage, contraintes de croissance, densité radiographique, retrait, anatonie.

\section{Introduction}

En raison de l'intérêt des échantillons non destructifs du type carottes de sondage pour étudier la qualité du bois, Pol.ge \& Thiercelin (1979) ont essayé d'apprécier les contraintes de croissance du hêtre à partir de la mesure du diamètre tangentiel d'une carotte de sondage.

De même, Ferrand (1981 et 1982) a montré la validité de cette méthode sur hêtre, peuplier et eucalyptus, ainsi que Okuyama (1983) sur peuplier. Ferrand (op. cit.) a remarqué que le bois de tension accompagne toujours les fortes contraintes de croissance.

Il est probable que la variation de quelques-unes des propriétés physiques du bois du cour vers l'écorce soit influencée par la présence de contraintes de croissance.

Le but de cette note est d'ćtudier sur des échantillons du type carottes de sondage la variation du cour vers l'écorce du retrait, en liaison avec différentes carac- 
téristiques anatomiques comme les gros rayons ligneux, leur nombre et leur densité, les caractéristiques des fibres et l'angle des microfibrilles, d'une part sur le bois de hêtre normal, et d'autre part sur le bois de hêtre à fortes contraintes de croissance.

\section{Matériel et méthode}

Deux hêtres dominants d'environ 200 ans de la forêt domaniale du Donon (Alsace) ont été choisis, l'un à bois normal, l'autre présentant un bois à fortes contraintes de croissance $\left(\varepsilon_{I_{t}}=3000 \times 10^{-6}\right)$. Le choix des arbres a été fait sur un lot d'une vingtaine d'arbres abattus dans la forêt mentionnée. Pour simplifier la nomenclature, le premier sera appelé " hêtre normal » et le deuxième «hêtre éclaté 》 (puisqu'il a éclaté un quart d'heure après labattage).

Le retrait a été exprimé comme la différence du diamètre de la carotte entre l'état saturé et l'état sec à l'air divisée par le diamètre à l'état sec à l'air (12 p. 100 d'humidité).

La mesure des diamètres a été faite avec un micromètre digital à 1 fum près, spécialement construit par J.R. PERrin à la Station de Recherches sur la Qualité des Bois (Perrin \& Ferrand, 1984).

Le retrait a été calculé à partir des valeurs ainsi mesurées pour 12 cycles successifs de saturation-séchage (de la saturation totale, obtenue par immersion sous vide à 20 " $\mathrm{C}$, à l'état sec à l'air à 12 p. 100 d'humidité).

Une autre caractéristique étudiée était l'infradensité. Le calcul a été fait à partir des valeurs des masses des carottes à l'état anhydre $\left(m_{6}\right)$ et à létat saturé $\left(m_{s}\right)$ par la formule bien connue de KeYLWFrTh (1954).

Les observations faites au niveau macroscopique sur des rondelles nous ont montré que la rupture engendrée par les contraintes de croissance dans le plan transversal (TR) se produisent selon les rayons ligneux. Pour cette raison, nous nous sommes attaché à leur étude. Ainsi, dcux sćries de mesures ont été faites :

Au niveau macroscopique, le nombre de gros rayons (visibles à l'oeil nu sur la rondelle prise à proximité de l'endroit du prélèvement de la carotte) a été rapporté à la surface totale de la rondelle.

A un niveau plus lin, la densité des gros rayons a été mesurée selon la méthodologie de Keller \& ThIlzcelin (1975) à partir de radiographies aux rayons $\mathrm{X}$ de coupes de $1,5 \mathrm{~mm}$ d’épaisseur (fig. 1) prélevées dans chaque carotte diviséc en 12 zones radiales. Lorsque les échantillons sont radiographićs radialement, les rayons apparaissent dans le plan LT comme des zones sombres. Pour obtenir la radiographie, les échantillons ont été placés sur le film à $2,50 \mathrm{~m}$ de la source de rayons $X$ et irradiés 10 minutes sous une tension de $12 \mathrm{kV}$ et une intensité de $20 \mathrm{~mA}$.

Les profils densitométriques ont été obtenus avec un microdensitomètre Joyce en utilisant une fente exploratrice large (largeur : $0,404 \mathrm{~mm}$ et hauteur : 1,34 mm, ce qui donne $0,54 \mathrm{~mm}^{2}$ ). Dans cette modalité, seuls les très gros rayons ligneux sont bien individualisés sur les graphiques. 

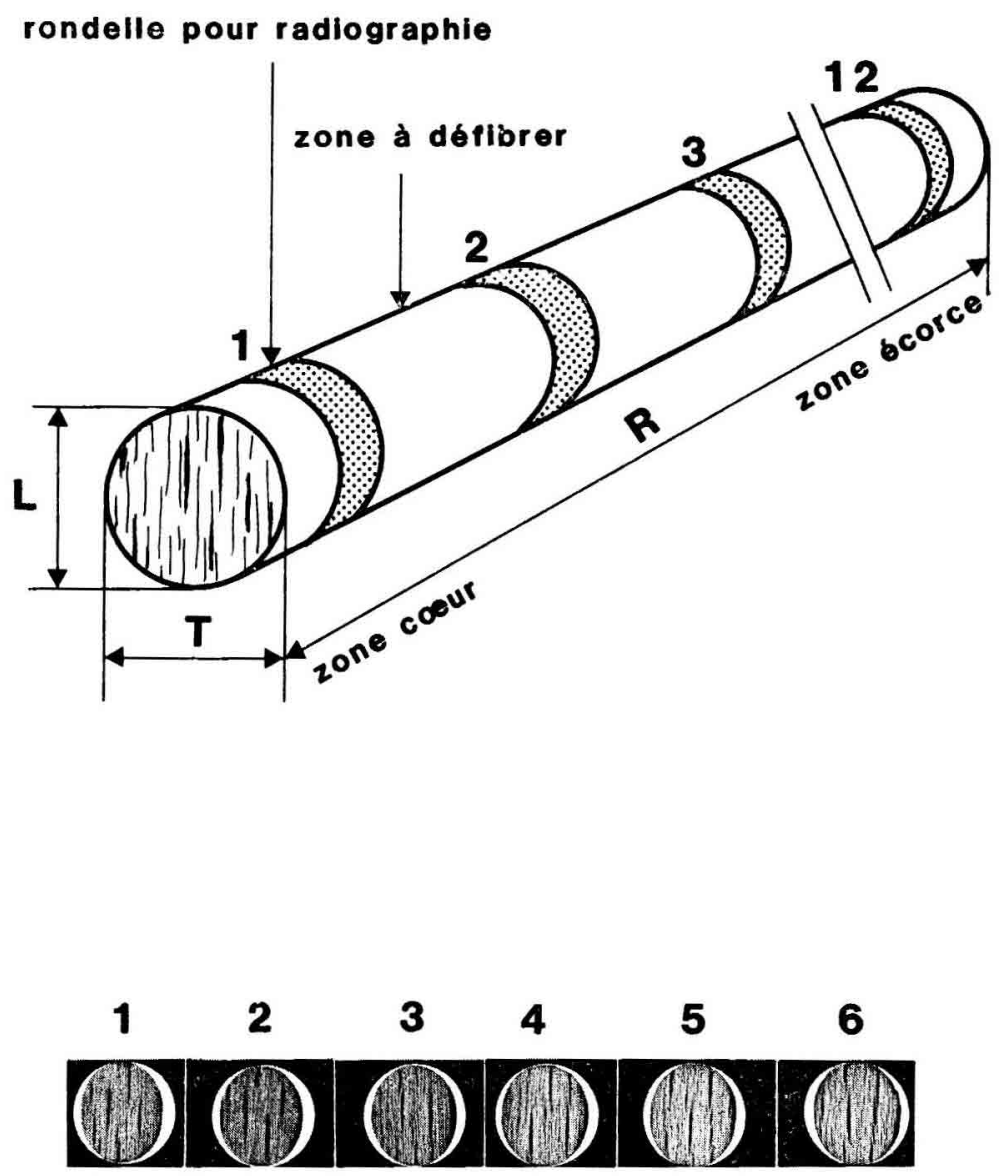

zone coeur

zone écorce
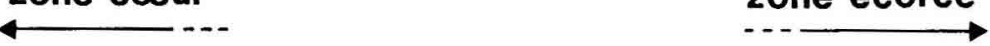

FIG. 1

Radiographie des 7 zones dans le plan tangentiel d'une carotte de sondage.

Les gros rayons ligneux sont observables comme des zones sombres.

Radiographic image of 7 zones in tangential plane of an increment core.

The arrangement of big rays are viewed in dark zones.

Langle des microfibrilles selon la méthode de diffraction aux rayons $X$ a été mesuré sur des clichés (fig. 2) obtenus à l’aide d’un générateur Philips équipé d’une anticathode de cuivre $(40 \mathrm{kV}$ et $13 \mathrm{~mA}$ ) sur des échantillons de $1 \mathrm{~mm}$ d'épaisseur. L'étendue angulaire de la tache équatoriale a été mesurée avec un microphotomètre Zeiss. La valeur de langle des microfibrilles est mesurée par rapport à l'axe longitudinal de la cellule ligneuse selon la méthode décrite par Boyd (1977).

Les caractéristiques des fibres ont été mesurées sur un microscope (grossissement 250). La microcuisson des fibres a été faite selon la méthodologie proposée par JaNIN 
(1983) pour toutes les zones radiales des carottes restantes après la préparation des échantillons pour l'analyse densitométrique des rayons ligneux.

Le diamètre et la longueur de 30 fibres de chaque zone ont été mesurés. Toutes les caractéristiques présentées ici ont été interprétées en fonction du numéro du cerne, compté du coúr vers l'écorce (numéro qui indique aussi l'âge de l'arbre).

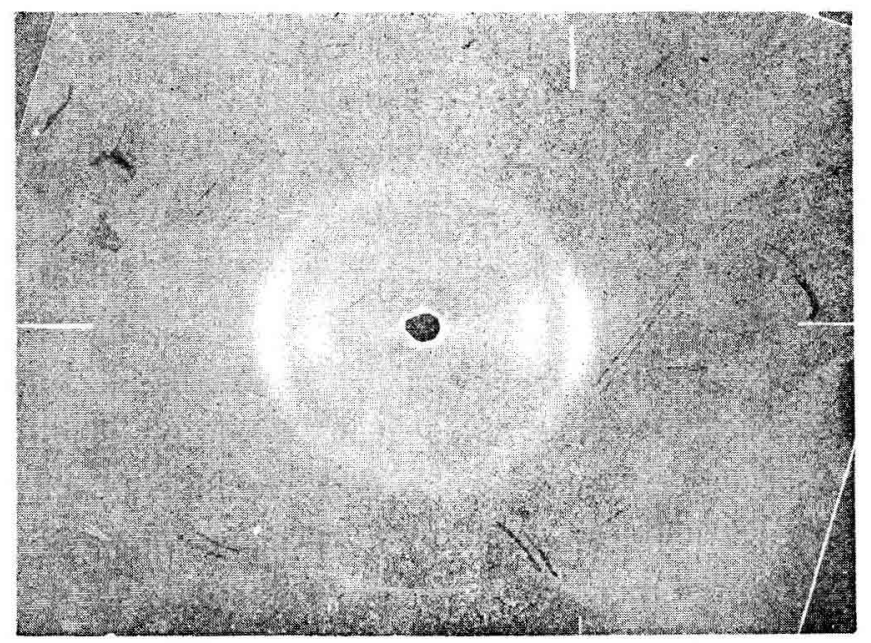

FIG. 2

Image de diffraction des rayons $X$ pour le bois de hêtre, côté coutur. utilisée pour la détermination de l'angle des microfibrilles (photo Dupont).

$X$-ray diffraction diagram of beech in heart zone, used for microfibril angle measurement.

\section{Résultats}

Dans ce qui suit, nous analyserons la variation du coeur vers l'écorce des caractéristiques suivantes : l'infradensité, le retrait, la largeur de cerne, les rayons ligneux. les dimensions des fibres.

\section{Ainsi :}

L'infradensité du bois normal (moyenne : $442 \mathrm{~kg} / \mathrm{m}^{3}$ ) est inférieure à celle du bois à fortes contraintes de croissance (moyenne : $491 \mathrm{~kg} / \mathrm{m}^{3}$ ). Le test $\mathrm{t}$ montre une différence significative à 0,1 p. $100(t=6,74 * *)$. La variation de l'infradensité du cœur vers l'écorce est montrée dans la figure 3.

Le retrait longitudinal (fig. 4) mesuré après le premier cycle de saturation pour le bois éclaté varie de $-0,15$ p. 100 à $+0,5$ p. 100 , tandis que pour le bois normal étudié ici, il est pratiquement toujours négatif (à l'exception de la zone de bois juvénile). Le bois à fortes contraintes de croissance présente un retrait longitudinal positif jusqu'au $20^{\circ}$ cerne. Entre le cerne 30 et 50 , le retrait longitudinal est négatif, 
et il change de signe avant le cerne 60 , pour se maintenir positif jusquà la fin de la vie de l'arbre.

Lal variation du retrait longitudinal moyen après 12 cycles de saturation est présentéc dans la figure 5. Le bois normal se stabilise après environ 8 cycles de saturation, tandis que le bois éclaté continue à présenter des variations importantes, comprises cntre $-0,3$ p. 100 et $+0,3$ p. 100 .

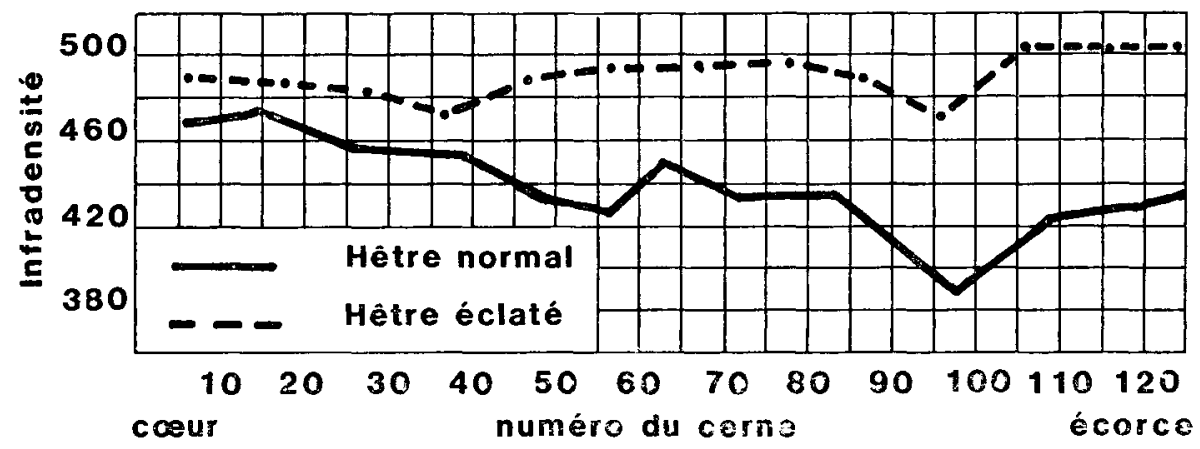

FIG. 3

Variation de linfradensité du coeur vers l'écorce.

Specific gravity variation from heart to the bark.

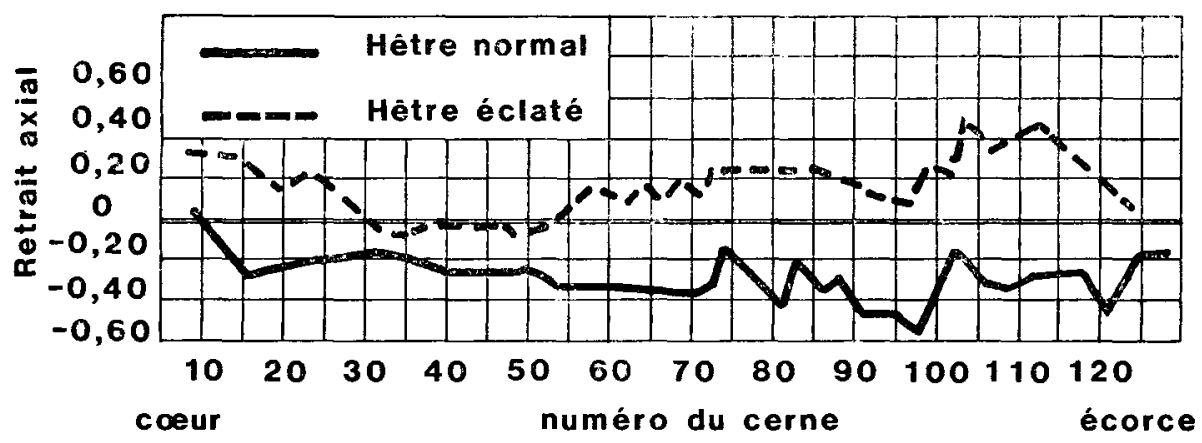

FIG. 4

Variation du retrait longitudinal du coutur vers l'écorce après le premier cycle de saturation. Longitudinal shrinkage from heart to the bark after the first saturation cycle.

Le retrait tangentiel est semblable pour le bois normal et éclaté (statistiquement, il ñy a pas de différence significative). Son domaine de variation est de 8 à 14 p. 100 , avcc une moyenne de 11,5 p. 100 pour le bois éclaté et de 11,4 p. 100 pour le bois normal. 


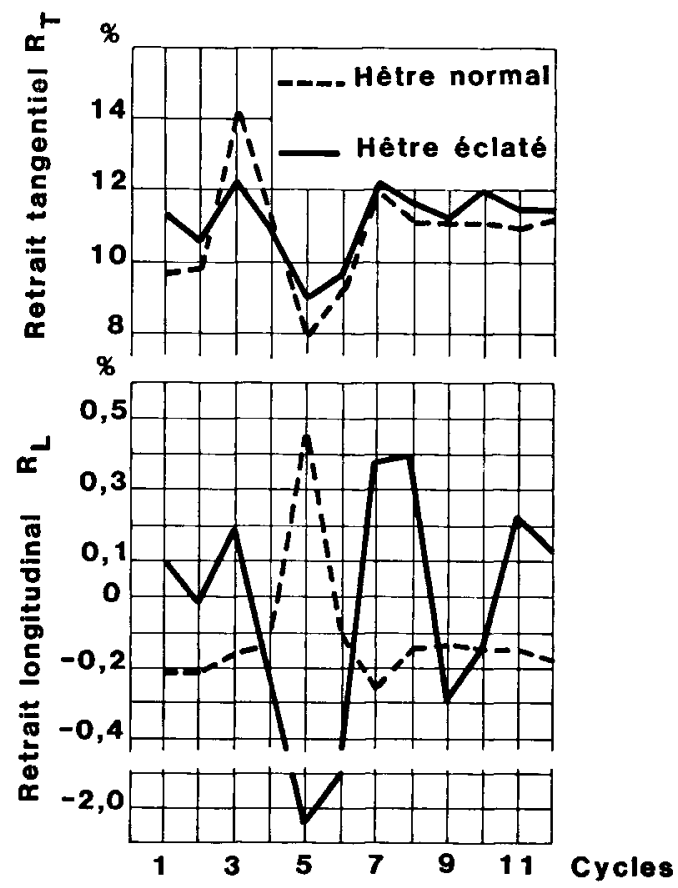

FIG. 5

Variation du retrait (longitudinal et tangentiel) en fonction du nombre de cycles de saturation. Valeurs moyennes par carotte.

Relationship between shrinkage (longitudinal and tangential) and number of saturation cycles. Average values on cores.

La largeur de cerne est un peu supérieure pour le bois éclaté $(1,95 \mathrm{~mm}$ en moyenne contre $1,61 \mathrm{~mm}$ en moyenne pour le bois normal), mais la différence n'est pas significative du point de vue du test $t$.

Les gros rayons ligneux (fig. 6) sont en nombre presque identique pour les deux bois jusqu'au cerne 50 . Entre les cernes 50 et 60 , le bois éclaté présente une augmentation importante de leur nombre au $\mathrm{cm}^{2}$ de 2 à 4 . Ensuite, il augmente lentement (de 5 à 7) jusqu'à la fin de la vie de l'arbre. Dans le bois normal, le nombre de gros rayons augmente lentement, sans dépasser $5 / \mathrm{cm}^{2}$.

La densité radiographique des gros rayons est en moyenne de $914 \mathrm{~kg} / \mathrm{m}^{3}$ pour le bois normal et $720 \mathrm{~kg} / \mathrm{m}^{3}$ pour le bois éclaté (fig. 7). Cette différence d'environ 30 p. 100 de densité $(t=9,69 * * *)$ peut expliquer la propagation facile de la rupture du bois à fortes contraintes de croissance dans les gros rayons ligneux.. 


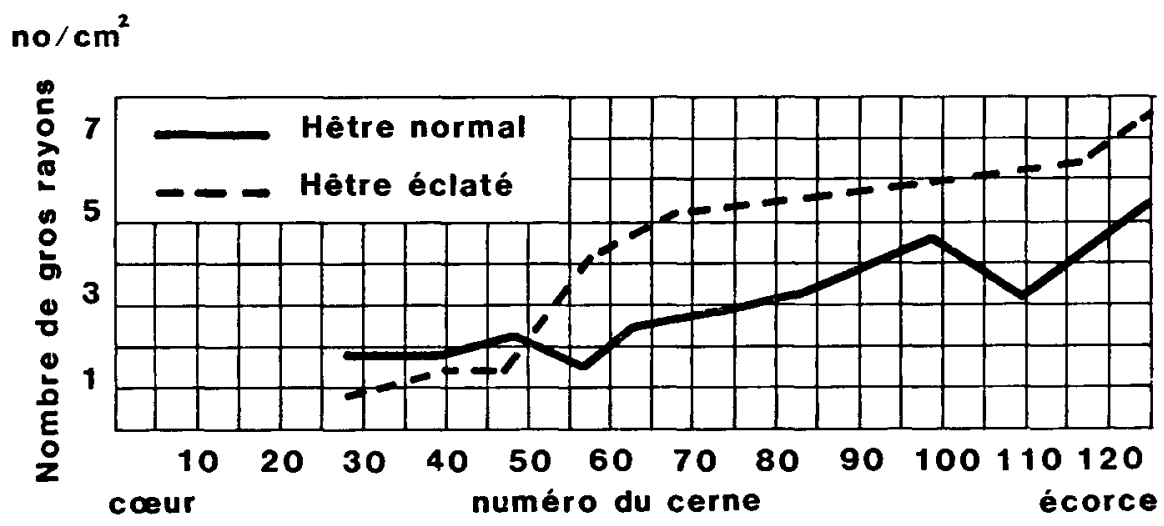

FIG. 6

Variation du nombre de gros rayons ligneux du caur vers l'écorce.

Variation of number of big rays from the heart to the bark.

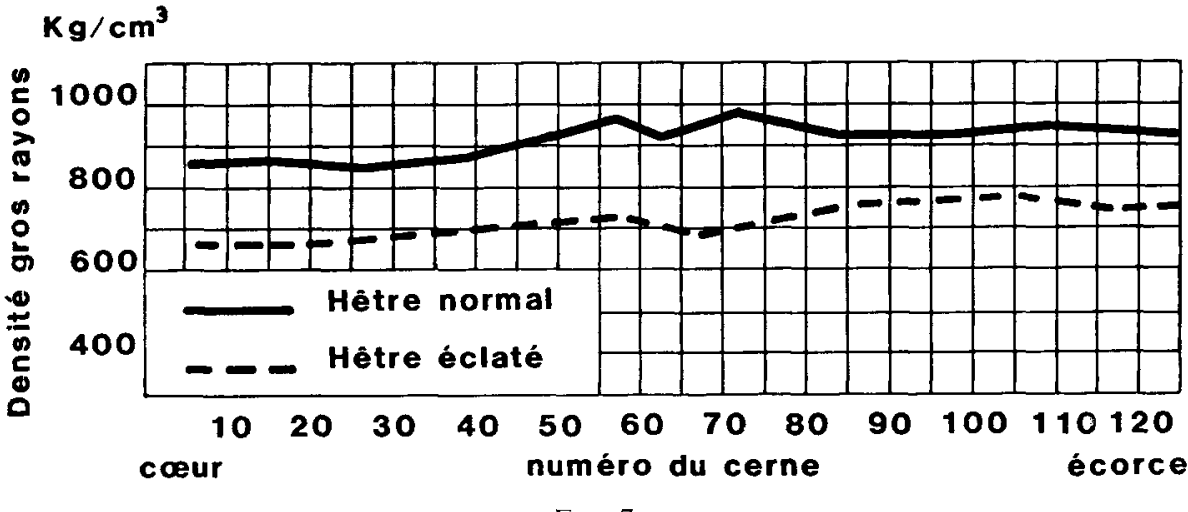

FIG. 7

Variation de la densité radiographique des gros rayons ligneux du coutur vers lécorce.

Variation of the radiographic density of big rays from the heart to the bark.

Les fibres du bois éclaté ont un diamètre (d) compris entre 10 et 15 fm et une longucur l comprise entre 0,8 et $1,2 \mathrm{~mm}$ (moyenne : $1,10 \mathrm{~mm}$ ). La variation du rapport $\mathrm{l} / \mathrm{d}$ est montrée dans la figure 8 . Par ailleurs, nous pouvons délimiter plusieurs domaines de variation du rapport $1 / \mathrm{d}$ en fonction de l'âge de l'arbre (exemple : cernes 5 à $50, \mathrm{l} / \mathrm{d}=60 \ldots 80$ ); cernes 50 à $60, \mathrm{l} / \mathrm{d}=80$ à 95 ; cernes 60 à 80 , $1 / \mathrm{d}=100 \ldots 120$; cernes supérieurs à $80,1 / \mathrm{d}=120$, pratiquement constant). 

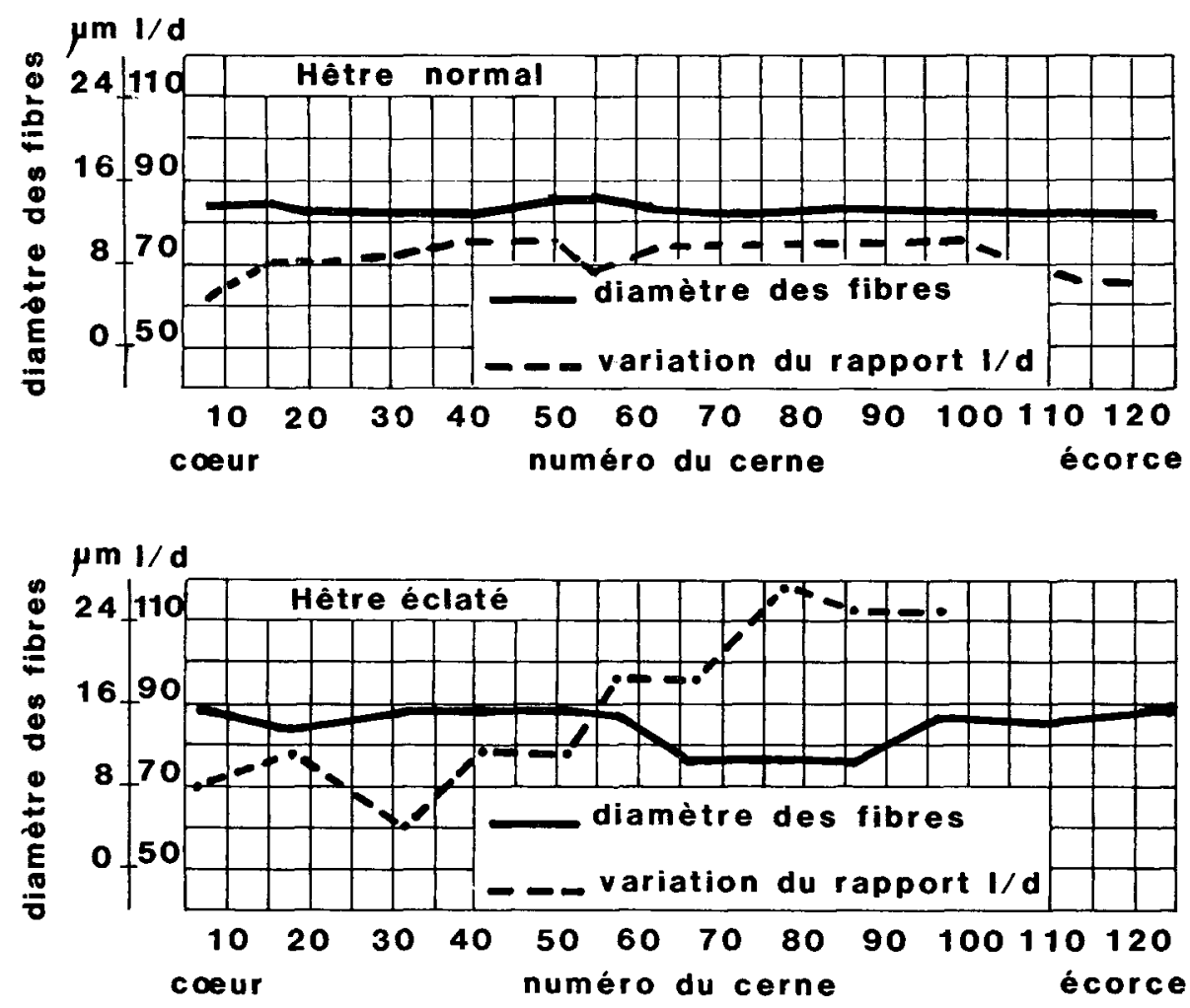

Fig. 8

Vartation des caractéristiques des fibres du caur vers l'ecorce.

Variation of fibers characteristics from the heart to the bark.

a) Bois normal - Normal wood.

b) Bois éclaté - High stress wood.

Pour le hois normal, la moyenne de la longueur des fibres est de $1,01 \mathrm{~mm}(0,93$ à $1,11 \mathrm{~mm}$ ) et le diamètre est de 14.35 fum $(13.81$ à 14,96). Le rapport $/ / d$ en fonction de l'âge est pratiquement constant. I.e domaine de variation est de 64 à 73 .

En résumé, nous pouvons affirmer que, pour le cas étudié, la longueur des fibres du bois éclaté est plus grande que celle du bois normal. Le bois à fortes contraintes de croissance se caractérise par des fibres plus longues que celles du bois normal (en moyenne $1,10 \mathrm{~mm}$ pal rapport à $1,00 \mathrm{~mm}$ ), et par un grand coefficient de souplesse, très variable du cour vers l'úcorce $(1 / \mathrm{d}=60 \ldots 80 \mathrm{du}$ côté cour, et $\mathrm{l} / \mathrm{d}=120 \mathrm{du}$ côté écorce pour le bois éclaté, tandis que $1 / \mathrm{d}=77$ en moyenne pour le bois normal).

La valeur moyenne de langle des microfibrilles est de 18,3 degrés pour le bois normal et de 16,8 degrés pour le bois éclaté. La différence entre ces deux moyennes est significative à 1 p. 100 (fig. 9). I.a décroissance lente de l'angle des microfibrilles 
du coeur vers l'écorce (de 15 à 11 degrés) observée par OKano et al. (1972) dans le bois final de Fagus crenata peut être comparée avec la décroissance de l'angle des microfibrilles du bois de hêtre à fortes contraintes de croissance (de 17 à 15 degrés). Pour le bois normal, l'angle des microfibrilles est pratiquement constant (18 degrés) du cour vers l'écorce.

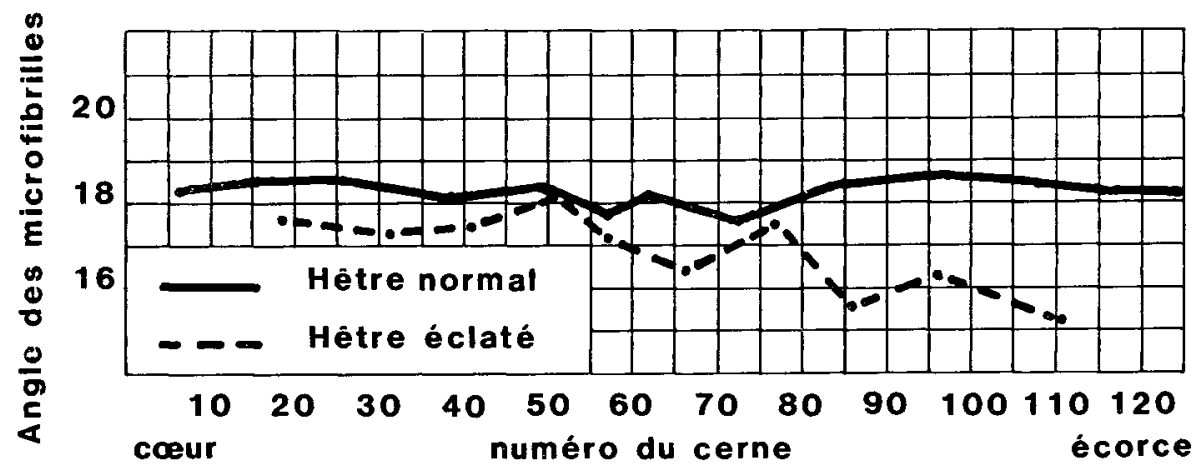

FIG. 9

Variation de l'angle des microfibrilles mesurée da caur vers l'acorce. Variation of microfibril angle from the heart to the bark.

De toutes ces constatations, nous pouvons déduire que le bois à fortes contraintes de croissance étudié ici se caractérise par une infradensité, un retrait longitudinal et un nombre de gros rayons par $\mathrm{cm}^{2}$ légèrement supérieurs aux caractéristiques correspondantes du bois normal. En revanche, la densité des rayons ligneux et langle des microfibrilles du bois à fortes contraintes de croissance sont plus faibles que ceux du bois normal.

\section{Conclusion}

Létude fine, cerne par cerne, de quelques propriétés physiques du bois de hêtre à fortes contraintes de croissance par comparaison avec du bois normal qui a poussé dans des conditions de station pratiquement identiques, montre qu'il est possible de discerner des différences entre les deux sur des échantillons non destructifs du type carottes de sonclage. Elles sont observables sur les caractéristiques suivantes : l'infradensité, le retrait longitudinal, la densité des gros rayons ligneux, les fibres (le rapport longueur/diamètre) et l'angle des microfibrilles.

Puisqu'il ne s’agit que d'un nombre restreint d'arbres étudiés, sur lesquels nous avons fait beaucoup de mesures pour confronter plusieurs méthodes d'appréciation de la qualité du bois, les résultats de cette note devraient être retenus surtout en termes de méthodologie. 


\section{Remerciements}

L'auteur remercie M. le Professeur Gaston Dupont de l'Université de Nancy I (Laboratoire de Biophysique) pour son concours lors de la mesure de l'angle des microfibrilles par la méthode de diffraction aux rayons X, et M. Michel Neveux, technicien à la "Qualité Bois», pour son aimable participation dans la préparation des échantillons pour la radiographic et pour les mesures des caractéristiques des fibres.

\section{Summary \\ Some physical properties of beech wood from hith growth stress trees measured on increment cores}

The relationship between shrinkage and density of the big rays, the characteristics of fibers, the microfibril angle and the basic gravity of beech wood having high growth stresses were studied on increment cores.

The detailed study, ring by ring from the heart to the bark, shows that in this experiment the high growth stress wood is characterized by a slightly higher basic density, longitudinal shrinkage, and number $/ \mathrm{cm}^{2}$ of big rays compared to normal wood.

On the other hand the radiographic density of big rays and the microfibril angle of beech wood having high growth stresses are lower than that of normal wood. elements.

Key woras : Increment cors, growth stress, X-ray density, shrinkage, amatomical

\section{Références bibliographiques}

BoYD J.D., 1977. Interpretation of X-ray diffractograms of wood for assessments of microfibril angles in fiber cell walls. Wood Sci. Technol., 11, 93-114.

FERRAND J.Ch., 1981. Recherches des solutions pratiques à apporter aux problèmes posés par les contraintes de croissance des arbres forestiers. Thèse de Docteur-Ingénieur en Sciences du Bois, I.N.P.L., Nancy, 11 décembre.

FERRAND J.Ch., 1982. Etude des contraintes de croissance. $1^{\text {re }}$ partic: Méthode de mesure sur carottes de sondage. Amn. Sci. For., 39 (2), 109-142.

Ferrand J.Ch., 1982. Etude des contraintes de croissance 2" partic : Variabilité en forêt des contraintes de croinssance du hêtre. Ann. Sci. For., 39 (3), 187-218.

Ferrand J.Ch., 1982. - Etude des contraintes de croissance $3^{\circ}$ partie : Eacalyptus delegatensis et Eucalyptus nitens : Influence de la sylviculture et de la station. Ann. Sci. For., 39 (4), 355-378.

Ferrand J.Ch., 1982. Growth stresses and silviculture of Eucalyps. Aus. For. Res., 13, 75-81.

JaNIN G., 1983. Microtests papetiers, microcuisson, microclassage, microraffinage, mesure automatique de la longueur des fibres. Thèse de Docteur d'Etat ès-Sciences, I.N.P., Grenoble, 5 mai.

Keller R., Thiercelin F., 1975. Influence des gros rayons ligneux sur quelques propriétés du bois de hêtre. Ann. Sci. For., 32 (2), 113-129.

Keylwrikth R., 1954. Studien über die Anwendung mathematischstatistichen Methoden in Holzforschung und Holzwirthschaft. Holz als Roll-und Werkstoff, 12 (3), 77-83.

Kumit:R H., 1959. Studien über Wachstumsspannungen des Holzes. Erste Mitteilung. $\mathrm{Holz}$ als Roh-und Werkstoff, 17 (1), 1-9.

Okano T., Onuki S., Miral S., 1972. On the distribution of the mean micellar angle and the fiber length in the trunk of Bune wood. Journal of the Japanese Wood Research Society, 18 (12), 583-586.

OkuYama T., 1983. Report on some experimental works on growth stress in wood. Mesure locale des contraintes de croissance près du cambium. Centre Technique du Bois, Paris, Action. de Recherche $n^{\circ} 208$.

Perrin J.R., Ferrand J.Ch., 1984. Alutomatisation des mesures sur carottes de sondage de la densité du bois, de son retrait et des contraintes de croissance. Ann. Sci. For., 41 (1), 69-96.

POlge H., Thiercelin F., 1979. Growth stress appraisal through increment core measurements. Wood Science, 12 (2), 86-92. 